\title{
Knowledge, Treatment-Seeking, and Socioeconomic Impact of Malaria on the Essequibo Coast of Guyana
}

\author{
Christopher M. Booth*, M.D. and J. Dick MacLean ${ }^{\dagger}$, M.D., F.R.C.P.(C.)
}

\begin{abstract}
The study was conducted to provide insight into malaria control efforts in Guyana, and to identify areas to emphasize in future educational campaigns. To do this, a community-based survey of knowledge, treatment-seeking patterns, and socio-economic impact of malaria was conducted at four outdoor markets in Region 2 Guyana. One hundred and eight individuals between the ages of 16 and 65 who had a malaria infection in the previous twelve months were interviewed. Within the study population, $94 \%$ identified mosquitoes as being the source of malaria infection. More than $70 \%$ of respondents identified fever, headache and chills as symptoms of malaria. Sixty percent of individuals incorrectly believed that women could not be treated with antimalarials when pregnant or they risked spontaneous abortion or congenital defects. Most individuals (76\%) used bed nets although very few nets were chemically treated. Mean delay in presentation to a health clinic was 6.3 days. Use of the official health care sector was high $(96 \%)$ and relatively few individuals $(15 \%)$ self-treated with antimalarials. Compliance with antimalarial regimens was also found to be relatively good $(92 \%)$. Cost of treatment was significantly higher among those who used private clinics (US\$ 13.74) than those who used public clinics (US\$ 0.96) $(p<0.001)$. The good level of knowledge of malaria may be due to the relatively high literacy rate and level of education in Guyana. The fact that public clinics in Guyana provide treatment and antimalarials at no cost may explain the relatively high use of the official health sector, low levels of self-treatment, and good compliance.
\end{abstract}

\section{INTRODUCTION}

Malaria is a vector-borne disease affecting 500 million people annually. $41 \%$ of the world's population reside in endemic regions, and the disease accounts for approximately 2 million deaths each year (1). Malaria infection is caused by the Plasmodium parasite which is transmitted to humans by the Anopheles mosquito. Plasmodium falciparum and Plasmodium vivax are both

\footnotetext{
* To whom correspondence should be addressed: School of Medicine, Queen's University, Kingston, Ontario, Canada K7L 3N6; Email: 4cmb@qlink.queensu.ca.

$\dagger$ Center for Tropical Diseases, McGill University, Montreal, Quebec, Canada.
}

present in South America. P. falciparum causes cerebral malaria, and accounts for the vast majority of malaria mortality worldwide.

Since the development of the Global Malaria Control Strategy by the World Health Organization in 1992, emphasis in malaria control has shifted from vector eradication to increased case detection and treatment of malaria (2). In addition to the official health care sector, the success of this approach depends significantly upon the general population. Consequently, malaria morbidity and mortality, and the problem of antimalarial resistance, are becoming increasingly dependent upon the knowledge and practices of affected individuals in malaria endemic countries. 
Previous studies have reported that the knowledge of etiology, symptoms and treatment of malaria is generally poor in the Americas and elsewhere in the world (3-9). Furthermore, preventive measures (i.e. chemoprophylaxis, mosquito netting) are not widely used in these populations $(4,5,9,10)$. Self-treatment of malaria (often with antimalarials) is widely practiced throughout the world $(3,5,6,8,10-16)$. Among those who do seek medical attention, there is often a delay of many days before presentation $(3,6)$.

Economics also contribute to the challenges of malaria control. Malaria-endemic countries are largely developing nations with limited financial resources. The morbidity associated with malaria and the cost of treatment can be important burdens. Economic limitations contribute to the difficulties in malaria control at the governmental level and for individuals (3,5,8-10,13,16-19).

Guyana is located along the north-eastern coast of South America. It is an English-speaking country with an area of $215000 \mathrm{~km}^{2}$ and a population of approximately 770 000. The population includes people of East Indian $(49.5 \%)$ and African descent $(35.6 \%)$ together with Amerindians (6.8\%) and people of other ethnic groups $(8.1 \%)(20)$. Sugar, rice and bauxite production account for a significant portion of the country's gross domestic product (GDP). Average monthly earnings are approximately US\$ 63. The majority of the population has received a minimum of six years of formal education and the literacy rate is estimated to be greater than $80 \%(20,21)$.

The Anopheles darlingi malaria vector was largely eradicated within Guyana by 1951 through widespread DDT spraying and the distribution of chloroquinecontaining salt. Low rates of malaria infection persisted until the mid-1970s when the incidence began to increase (22). In 1998 there were 39370 reported cases of malaria $(58.5 \%$ P. falciparum, $41.5 \% \quad P$. vivax) in Guyana with a calculated Annual Parasite Index (API) of 52.1 (23). Guyana and Suriname have the highest rates of malaria infection in the Americas (24). Malaria was reported to be the second leading cause of morbidity in Guyana in 1996 (20). Although more than 80\% of Guyana's population lives along the coastal plain, the majority of malaria infections originate in the interior within the mining and logging communities (20-22).

The current malaria control program in Guyana involves passive and active case detection together with DDT spraying of dwellings in high risk areas (23). In 1998 the national budget for health services was US\$21.3 million (approximately 3.4\% GDP) of which US\$ 640 093 was allocated for malaria control $(20,23)$. Guyana is known to have chloroquine-resistant $P$. falciparum, and cases of resistant $P$. vivax have also been reported $(25,26)$.
The aim of this community-based descriptive study was threefold. We sought to describe the knowledge of etiology and symptoms of malaria in a study population in Guyana. Furthermore, we examined the treatmentseeking patterns of this population, and quantified the socioeconomic impact of malaria infection on the affected individual. There has not been an abundance of research in this area within the Caribbean region. Thus, it was felt this study would provide insight into the efficacy of the current malaria control program in Guyana, identify targets for future educational campaigns, and provide guidance for existing programs in the Caribbean, and elsewhere in the world. Region 2 was chosen as the study site because it has an aboveaverage incidence of malaria, and it has many schools and public markets from which a future educational campaign could be launched (23).

\section{METHODS}

\section{Study area and population}

The study was undertaken in Region 2, Guyana (population 43 139). Region 2 (commonly referred to as the Essequibo Coast) is one of ten regions in Guyana and has the 6th highest incidence of malaria in the country (API 84.6) (23). The $3800 \mathrm{~km}^{2}$ region extends along the Atlantic Coast of Guyana to west of the capital city Georgetown.

The population of Region 2 is English-speaking. It is a rural region, with the majority of individuals living in small towns along the coast. Agriculture, mining and commerce are the main sources of employment in the region.

Region 2 is served by several different levels of a state-funded health care system. There is one district hospital which offers a full range of diagnostic and medical services and there is one regional hospital which offers basic medical and surgical services. There are 11 health centers throughout the region which provide basic primary healthcare. There are also three private clinics within the region. Approximately eight doctors and 60 nurses work in the region. Diagnostic tests for malaria are available only at the district hospital, the regional hospital and the private clinics.

\section{Study design and data collection}

This community-based, descriptive study was conducted from May 10 through June 61999. Individuals with a prior malaria infection were interviewed by a single investigator (C.B.) in the four largest outdoor markets of Region 2. Individuals were approached sequentially as they passed through the center of the market. Informed consent was obtained and individuals who had previously been infected with 
malaria were asked a series of open-ended and multiple choice questions. The study protocol was approved by the ethics committee of Queen's University Faculty of Health Sciences. The interview was conducted in English. There were 41 questions in total and the interview lasted approximately fifteen minutes. The questionnaire was divided into five sections: demographic characteristics, knowledge of malaria, prevention of malaria, number of previous malaria infections and treatment of most recent malaria infection. The original questions were modified after a pre-trial of ten individuals. (A copy of the questionnaire is available upon request.) Participants were read each question in the questionnaire. No compensation was offered to the respondents.

A total of 256 individuals with a previous malaria infection were interviewed. Only four individuals refused to participate in the study. 148 individuals were excluded because their malaria infection occurred prior to the past twelve months. This paper summarizes the results obtained for those between the ages 16 and 65 who had a malaria infection in the past year $(n=108)$.

\section{Data analysis}

All statistical analyses were performed with Microsoft Excel (Version 7.0, Microsoft Corporation) and Statistical Analysis System software (SAS Institute). The chi-square test was used to test for significance among groups with regard to proportional data. The Student's t-test (parametric data) and the Wilcoxon test (non-parametric data) were used to compare group means. All group means are reported with their standard deviation. A $p$ value of $<0.05$ was used to indicate statistical significance.

Sub-group analyses were performed for sex, education (primary school vs. more than primary school), occupation (mining vs. non-mining), marital status (married vs. single), number of past infections (first infection vs. multiple infections), initial treatment source (self vs. clinic) and clinic used (public vs. private).

\section{RESULTS}

\section{Characteristics of study population}

The study population included 108 individuals, 87 $(81 \%)$ men and $21(19 \%)$ women (Table 1$)$. The mean age was $34.5 \pm 12.2$ years. Mining was the most commonly reported occupation $(42 \%)$ with agriculture and commerce the next most common occupations ( $21 \%$ and $14 \%$, respectively). The majority $(61 \%)$ of the population had completed primary school (six years), with some individuals $(22 \%)$ reporting postsecondary education. There was no significant difference in education among sub-groups.
Table 1. Characteristics of the study population $(n=108)$

\begin{tabular}{lll}
\hline Characteristic & & Frequency or Mean \\
\hline \multirow{2}{*}{ Gender } & Male & $87(81 \%)$ \\
& Female & $21(19 \%)$ \\
Age (yr.) & Study population & $34.5(\mathrm{SD} 12.2)$ \\
& Male & $33.3(\mathrm{SD} 12.3)$ \\
& Female & $39.7(\mathrm{SD} 10.9)$ \\
Education & Primary School & $82(76 \%)$ \\
& $>$ Primary School & $26(24 \%)$ \\
Occupation & Mining & $46(43 \%)$ \\
& Agriculture & $23(21 \%)$ \\
& Commerce & $16(15 \%)$ \\
& Lumbering & $7(6 \%)$ \\
& Homemaker & $6(6 \%)$ \\
& Other & $15(9 \%)$ \\
Marital Status & Married & $57(53 \%)$ \\
& Single & $51(47 \%)$ \\
Household & No. of adults & $2.9(\mathrm{SD} 1.7)$ \\
& No. of children (<16 yr.) & $2.4(\mathrm{SD} 2.4)$ \\
\hline
\end{tabular}

\section{Knowledge of malaria}

Mosquitoes were identified in an open ended question as being the source of malaria by $94 \%$ of the population. This was not found to vary significantly with gender, occupation, education, or number of past malaria infections. Sixty-one percent of the population stated that mosquitoes were the only source of infection. The majority of the population $(77 \%)$ also believed that mosquitoes transmitted malaria from one person to another. Thirty-one percent of the population incorrectly believed that malaria can be contracted by drinking dirty water. This belief was not found to vary significantly with gender, occupation, education or number of past infections.

Fever $(100 \%)$, headache $(86 \%)$, chills $(69 \%)$, rigors $(55 \%)$, vomiting $(35 \%)$ and arthralgia $(30 \%)$, were all commonly reported symptoms of malaria (Figure 1). The majority of individuals (87\%) stated that antimalarial tablets were the appropriate treatment for malaria, while $18 \%$ of respondents felt that traditional bush medicine was the only way to cure malaria. Sixty percent of the population incorrectly believed that women could not be treated with antimalarials when pregnant. There was no significant difference in responses between men and women. Of those who believed that pregnant women could not take antimalarials, $55 \%$ felt that spontaneous abortion would occur and $31 \%$ felt that congenital defects would result.

\section{Prevention of malaria infection}

Very few respondents took any form of chemoprophylaxis. Twenty-six percent of the study population stated that they had taken malaria chemoprophylaxis in the past. Most of these individuals received the antimalarials from an employer but not a 


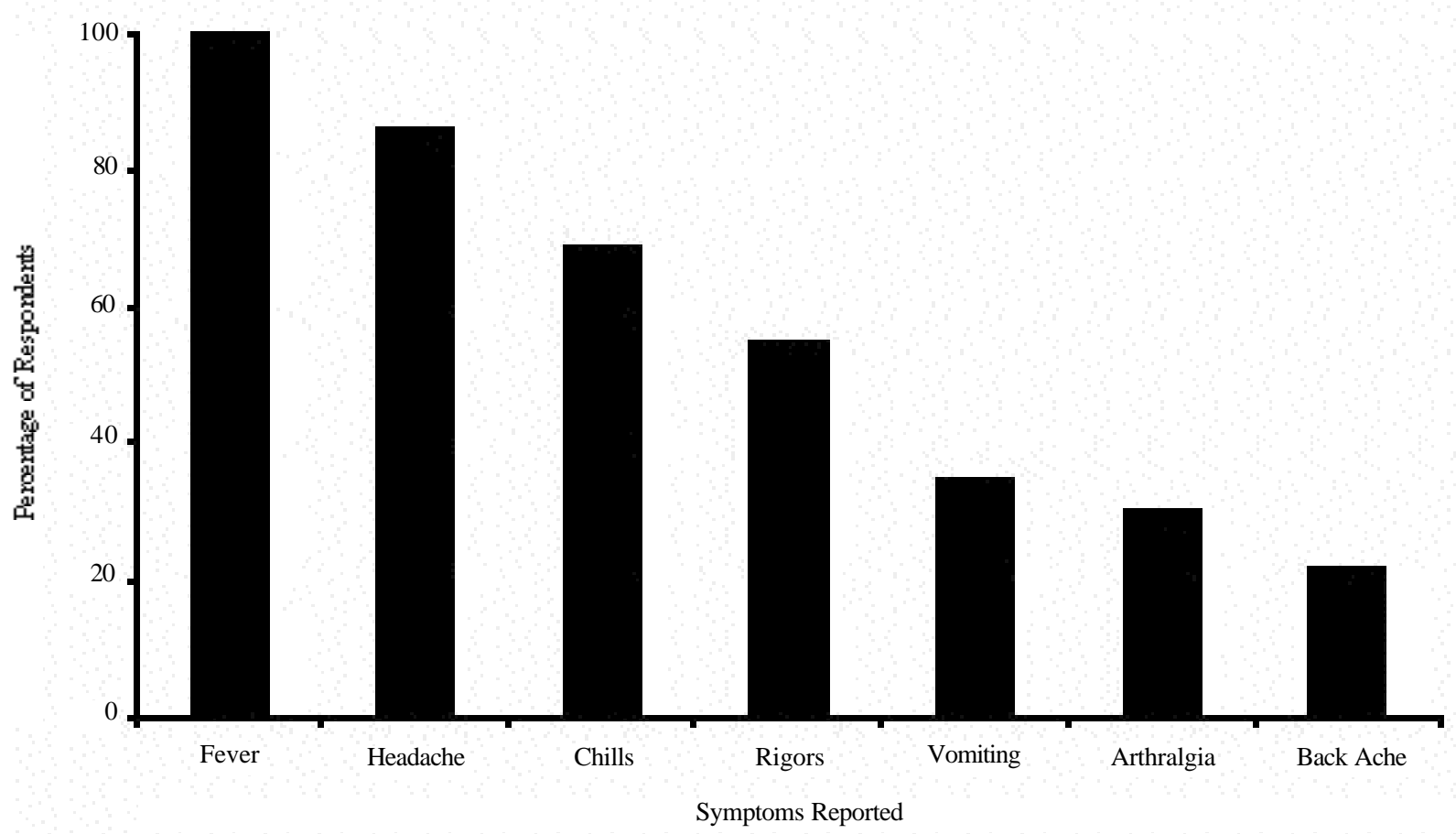

Figure 1. Symptoms of malaria as reported by subjects interviewed at outdoor markets $(n=108)$.

single individual reported taking chemoprophylaxis regularly. Men were more likely to have taken antimalarials for prevention than were women $(31 \% \mathrm{vs}$. $5 \%$, chi-square test, $p=0.01)$. Level of education, occupation, or number of past infections did not significantly influence use of chemoprophylaxis.

The majority of individuals $(76 \%)$ used a bed net but very few of these individuals (16\%) reported having their net chemically treated. Seventeen percent of individuals reported having their home sprayed with DDT in the past 12 months and a small proportion $(8 \%)$ of respondents reported having screens on their windows.

Traditional bush medicines, primarily herbal drinks, were commonly used to prevent malaria. Fifty-nine percent of the study population reported using traditional medicine to prevent contracting malaria. This practice did not vary significantly with gender, education, or occupation.

\section{Number of past malaria infections}

The mean number of past malaria infections was 12.1 \pm 16.4 (Table 2 ). Four individuals (4\%) reported having had greater than 50 previous infections. On average, men had significantly more infections than women $(13.7 \pm 17.7$ vs. $5.4 \pm 6.2$, Wilcoxon test, $p=0.047)$. Occupation and education, were not found to be significant factors in the number of past infections.

\section{Treatment of last malaria infection}

The mean time since the most recent infection was $6.7 \pm 4.6$ months. One third of the sample population self-treated with antipyretics before seeking treatment from the healthcare sector. Only $15 \%$ of the population self-treated with antimalarials. Ninety-six percent of the population eventually presented to a health care facility for a diagnostic blood smear and treatment. The mean delay to presentation was $6.3 \pm 10.5$ days. Delay to presentation was not found to vary significantly with gender, education, occupation, marital status, number of past infections, or first source of treatment.

$80 \%$ of individuals waited for longer than 1 day before presenting to the health care sector. Forty-one percent of this group said they had to wait for transportation to be available, $36 \%$ of the group waited because they were unsure about their condition, and $15 \%$ delayed seeking treatment because the symptoms were mild. Reasons for delay to treatment were not found to be significantly dependent upon gender, occupation, education, number of past infections or martial status. However, $76 \%$ of individuals (13 of 17) who had never been previously infected with malaria said they waited before seeking treatment because they did not think they had malaria, while only $28 \%$ of individuals who had had previous infections waited for this reason (chi-square test, $p<0.001$ ). 
Of the $104(96 \%)$ individuals who had a diagnostic blood smear for their most recent infection, $48 \%$ reported having $P$. falciparum and $45 \%$ reported having $P$. vivax malaria infections. Miners were less likely to have a diagnostic smear than non-miners (91\% vs. $100 \%$, chi-square test, $p=0.018$ ). Eighty-eight percent of individuals had a blood smear done at a public clinic, while $8 \%$ of the population had a smear at a private clinic. Significantly more women went to a private clinic than men $(19 \%$ vs. $6 \%$, chi-square test, $p=$ 0.048), and those who had never had a malaria infection were more likely to go to a private clinic than those who had previously had malaria $(24 \%$ vs. $5 \%$, chi-square test, $p=0.017$ ).

Treatment of the most recent malaria infection involved antimalarial tablets in $100 \%$ of the cases. Fourteen percent of individuals reported having an injection to treat the infection while several others had saline infusions, bush medicine or vitamins $(9 \%, 2 \%$ and $5 \%$, respectively). The most commonly prescribed antimalarials were quinine $(65 \%)$, primaquine $(47 \%)$, chloroquine $(26 \%)$, and sulfadoxine-pyrimethamine $(17 \%)$. Quinine was prescribed more frequently for $P$. falciparum (75\%) than $P$. vivax (53\%) (chi-square test, $p=0.021)$. Primaquine was prescribed more frequently for P. vivax (59\%) than P. falciparum (39\%) (chi-square test, $\mathrm{p}=0.037)$. There was no significant difference in treating $P$. falciparum $(21 \%)$ or $P$. vivax $(31 \%)$ with chloroquine. There is thought to be $100 \%$ chloroquineresistant $P$. falciparum in Guyana (25).

Compliance was assessed by asking the following three questions: How many days did you take antimalarials for? How many days were you told by your physician to take them for? and Did you finish all the tablets prescribed? Based on the answers to these questions, $92 \%$ of the population was found to have been compliant with their antimalarial regimen. There was no significant difference in compliance based on gender, occupation, education, number of past infections, marital status, quinine usage or public vs. private sector usage.

The nine non-compliant individuals were asked why they ended their treatment prematurely. The most common reason was adverse effects caused by the antimalarials ( 8 individuals). Three individuals stopped because they felt better and one individual reported that he simply forgot to continue with the treatment.

\section{Socioeconomic impact of last malaria infection}

The mean duration of symptoms was $13.2 \pm 13.0$ days. The mean number of lost employment days was $16.8 \pm 25.3$ (Table 3). Duration of symptoms and number of days off work was not found to vary significantly with public vs. private sector usage.
Table 2. Number of past malaria infections $(n=108)$

\begin{tabular}{llcc}
\hline Group & & \multicolumn{2}{c}{ No. Past Infections } \\
& & Mean & SD \\
\hline Study population & & 12.1 & 16.4 \\
Gender & Male $^{*}$ & 13.7 & 17.7 \\
& Female $^{*}$ & 5.4 & 6.2 \\
Occupation & Mining & 10.3 & 11.8 \\
& Non-mining & 13.4 & 19.8 \\
Education & Primary & 11.4 & 16.9 \\
& $>$ Primary & 14.1 & 14.7 \\
\hline
\end{tabular}

* Found to be significantly different (Wilcoxon test, $p=0.047$ ).

However, there was a significant difference in time off work between those who had $P$. falciparum $(24.5 \pm 33.2$ days) and those who had $P$. vivax malaria $(10.3 \pm 11.3$ days) (Wilcoxon test, $p=0.005$ ). The majority of individuals with $P$. falciparum described a prolonged period of weakness following their acute infection which prevented them from returning to work.

The mean cost of diagnostic blood smears and treatment was US $\$ 2.13 \pm 5.74$. Although most individuals $(91 \%)$ went to a public facility for a blood smear, not all of these people were able to obtain antimalarial treatment at the public clinic as public facilities periodically run out of supplies. Eighty-six percent of the population obtained their tablets from a public clinic, $8 \%$ from a private clinic, $7 \%$ from a pharmacy and $3 \%$ from a co-worker. The only significant factor influencing cost of treatment was whether an individual went to a public or private clinic. Those who went to a public clinic spent a mean US $\$ 0.96 \pm 4.05$, while those who went to a private clinic spent US $\$ 13.74 \pm 6.92$ (Wilcoxon test, $p<0.001$ ). Transportation was another sizable expense for the population. The mean cost of transportation for diagnosis and treatment was US \$7.22 \pm 14.38 .

\section{DISCUSSION}

\section{Knowledge of malaria}

The study population appears to be relatively knowledgeable of the etiology, symptoms, and treatment of malaria. The majority of the population correctly identified mosquitoes as the disease vector. Studies in Colombia and Nigeria found that the source of malaria, and means of transmission, were largely unknown $(7,9)$. In a survey of 300 women in Ethiopia, only $17 \%$ of participants identified mosquitoes as the source of malaria infection (5). However, not all studies have reported a poor understanding of etiology. Two studies in Guatemala reported that more than $90 \%$ of respondents recognized mosquitoes as the malaria vector $(12,27)$. In our study population 
Table 3. Employment lost (days) due to malaria infection $(n=108)$

\begin{tabular}{llll}
\hline Group & & \multicolumn{2}{c}{ Employment Lost (Days) } \\
& & Mean & SD \\
\hline \multirow{2}{*}{ Study population } & & 16.8 & 25.3 \\
Clinic visited & Public & 16.9 & 26.1 \\
& Private & 20.2 & 22.0 \\
Parasite species & P.falciparum $^{*}$ & 24.5 & 33.2 \\
& P.vivax* $^{*}$ & 10.3 & 11.3 \\
\hline
\end{tabular}

* Found to be significantly different (Wilcoxon test, $p=0.005$ ).

there is still a significant number of respondents who believe that malaria can be contracted by drinking dirty water. One of the studies in Guatemala also found this belief to exist in more than $50 \%$ of their study population (27).

Our study population demonstrated a good understanding of the symptoms of malaria (fever, headache, chills and rigors). This is of obvious importance in a malaria control program. Studies in Guatemala and Nigeria have also reported greater than $80 \%$ recognition of malaria symptoms $(9,11)$.

The majority of our respondents also correctly identified antimalarial drugs as the treatment of choice. Some individuals however, did state that traditional bush medicines could cure malaria. This belief was also reported in nearly $50 \%$ of a study population in Guatemala (12). Our results also show that most individuals believe that women should not use antimalarials when pregnant or they risked abortion. This misconception has been noted by other workers $(3,12)$. Foster has proposed that this belief exists in many countries because the bitter taste of chloroquine is associated with the bitterness of many traditionallyused abortificants (3). This poses a problem in the treatment of women with malaria. Nevertheless, the level of knowledge of malaria in our study population appears to be relatively good, and may be related to the relatively good level of education and literacy of the study population.

\section{Prevention of malaria infection}

Despite recognizing mosquitoes as the source of malaria, very few individuals reported using any consistent means of malaria prevention. While the majority of individuals did report using a bed net, very few nets had been chemically treated. One of the Guatemalan studies also reported a high rate of use (>70\%) of bed nets (27). In our study, no individuals reported using regular chemoprophylaxis. More than half of the cohort used bush medicines, (usually bitter drinks), to prevent infection. This is concerning as there is very little documented medical experience with these compounds. Despite the lack of proven preventive measures, our study population was much more likely to use bed nets than previously reported elsewhere. Studies in Zimbabwe, Kenya and Ethiopia all reported that less than $10 \%$ of respondents used a bed net $(4,5,9)$. This discrepancy may be explained by the relatively good understanding of malaria etiology in Guyana and that the Guyanese people may have more financial resources to obtain nets. The annual GDP per capita is greater in Guyana and Guatemala (US \$768 and US $\$ 1643$ ) than in Zimbabwe, Kenya and Ethiopia (US \$295, US \$271 and US \$162) (28). The fact that very few nets were chemically treated, suggests that this more effective approach needs to be more widely promoted by Guyanese health workers.

\section{Treatment of last malaria infection}

Self-treatment with antimalarials is reported to be widespread in malaria-endemic countries. Foster estimates that more than half of the world's antimalarials are consumed outside the public health sector (3). However, our results suggest that this is not the case in Guyana, as only $15 \%$ of respondents selftreated with antimalarials. While many individuals selftreated at home with antipyretics before seeking medical attention, $96 \%$ of the cohort did eventually present to a health care facility. Studies in Guatemala, Ethiopia, and Kenya found that more than $60 \%$ of individuals self-treated (usually with antimalarials) and did not seek medical attention $(5,10,11,15)$. Several workers have proposed that the reasons for selftreatment include financial cost and accessibility $(3,5,14)$. The low rate of self-treatment with antimalarials and the high level of use of the official health sector in our study may be related to the fact that treatment and medications are free at public clinics in Guyana and that the clinics are close to the homes of most affected individuals. The majority of the population in Region 2 lives within approximately ten $\mathrm{km}$ of a health clinic. A handful of other studies have also reported low rates of antimalarial self-treatment, although this appears to be the exception and not the norm $(3,4)$.

The mean delay to seeking medical attention in our population was comparable to results reported elsewhere $(6,8)$. This is of concern as a delay of even a few days can be fatal in malaria infection, especially in children (17). Many of our respondents delayed seeking medical attention because transportation was not readily available. Although there are many clinics in close proximity to the towns of Region 2, individuals working in the interior often have to wait until they return home to obtain health services. This was especially true among those who contracted malaria while working in the mines where access to health services is limited. 
More than one third of respondents waited for several days before seeking medical attention because they did not think they had a serious illness. Individuals who were infected with malaria for the first time were significantly more likely to delay for this reason than were those who had been previously infected. This suggests that symptom recognition may be a problem in individuals infected for the first time. Only a handful of individuals delayed for economic reasons.

In a review article, McCombie reported that most studies have found distance to health services and financial cost to be the reasons for delay in malaria treatment (6). While the length of delay in our study was comparable to other reports, the reasons for delay differed. We did not find that economic factors contributed to delay in treatment seeking. This may be explained in part by the fact that health services and treatment are free in Guyana.

Rate of completion of antimalarial treatment regimens is known to be poor throughout the malariaendemic regions of the world. Numerous studies have found compliance rates to be low, particularly in regimens involving quinine $(6,17,29-32)$. However, there have been reports in which compliance has been upwards of $70 \%(4,5,11,29,33)$. The limiting factor in almost all of these studies has been the fact that compliance has been assessed through subjective means such as patient interviews. Nevertheless, noncompliance is a widely acknowledged problem.

Non-compliance with antimalarials has been found to be related to four factors: adverse effects, early resolution of symptoms, saving of tablets for future use and inadequate dosing instructions (4-8,17,29$31,33,34)$. The relatively high level of compliance in our study may be attributed to the good level of knowledge of the respondents and the fact that the public health system provides treatment at no cost. Consequently there is no financial incentive to stop treatment prematurely in order to save tablets for future use. There were not found to be any statistically significant predictors of compliance. With the exception of one individual, all of the non-compliant individuals reported stopping their medication because of adverse effects. One third of individuals stopped prematurely because they felt better, and no individuals stopped in order to save the medication for future infections. Two other studies have reported compliance rates comparable to ours. In Guatemala, interviews with 3148 individuals found a compliance rate of $80 \%(11)$, while a compliance rate of $93.7 \%$ was found in a series of 300 women in Ethiopia (5). Antimalarials were provided at no cost to the population in Guatemala while most individuals in Ethiopia did pay for treatment. Although our compliance rate is likely to be artificially high due to recall bias (a recognized limitation in compliance assessments), the rate does appear to be greater than most other studies, in which the same bias would have been present.

\section{Socio-economic impact of last malaria infection}

The mean duration of symptoms and lost work due to malaria infection was approximately two weeks in our study. Individuals who had been infected with $P$. falciparum described a period of prolonged weakness and fatigue after the acute infection, causing them to miss significantly more work than those infected with $P$. vivax. Despite the widely held belief that private clinics offer better treatment, there was no significant difference in outcome noted between individuals who used public versus private clinics. However, individuals treated at private clinics paid significantly more for treatment than those treated at public clinics. Although treatment is free at public clinics, individuals occasionally had to purchase medication elsewhere if the clinic had run out of supplies. Transportation to seek medical care was also a significant expense for the study population. This is surprising as the majority of the population lives in close proximity to health services. During the interviews it was observed that many respondents went to clinics in towns other than their own. Some individuals even reported travelling $90 \mathrm{~km}$ to the capital city Georgetown, when there were malaria diagnostic and treatment services available minutes away from their home. Many of these individuals traveled to larger centers where they believed they would receive superior medical care. The expense associated with transportation and treatment for malaria can be significant, representing $10-20 \%$ of average monthly earnings.

In light of the significant economic cost of malaria, many governments are beginning to implement user fees for the treatment of malaria (3). In many countries, the population self-treats or uses private clinics because medications are frequently unavailable at public clinics $(5,14)$. Other authors report that selftreatment rates are high because of the inconvenience and inaccessibility of public health services $(3,5,16)$. Nevertheless there is a common theme throughout the literature: rising costs of malaria treatment result in decreased utilization of medical services, increased rates of self-treatment and decreased compliance $(3,5,8,9,16,17,19)$. The relatively low level of selftreatment and good compliance rates found in our study may be explained by the wide accessibility of public health services in Guyana and the fact that medications are free at public clinics. 


\section{Summary}

The results of our study must be interpreted in light of study limitations. Although our study was conducted in public outdoor markets the study population may not be representative of the general population. In particular, children, the population at greatest risk for morbidity and mortality is not represented. Furthermore, only $19 \%$ of our cohort were female. However, the majority of malaria infections in Guyana are known to occur in middle aged males working in the bush (20), and therefore our study population is representative of the burden of disease in Guyana. Another study limitation is the fact that questions about the most recent malaria infection rely on memory and recall of the respondents. This is especially true in the questions concerning compliance. However, the results found in our study may be compared to previous studies which were also limited by the same factors $(4-6,11,17,29-33)$. As our study only included survivors of malaria infection, our results may be biased and therefore should be applied to the general population with caution. Finally, as our sample included 108 individuals, small numbers in subgroups limited the power of our analyses.

In summary, the study population appears to be quite knowledgeable of the etiology, symptoms, and treatment of malaria. Patterns in treatment seeking and compliance suggest that the close proximity to health services and low cost are significant strengths of the Guyanese system. However, individuals working in the interior often have to wait several days to return to the coast where health services are widely available. The benefits of providing antimalarials at no cost (i.e. increased use of official health care sector, increased compliance and increased knowledge of etiology and symptoms) suggest that other countries should consider this as a means of decreasing malaria morbidity and mortality. In the study population there is a place for future education about prevention of malaria (especially bed nets), the benefits of early treatment and the treatment of pregnant women. More research is needed in the future to further investigate the role of economics in the treatment of malaria in Guyana.

\section{ACKNOWLEDGMENTS}

The authors gratefully acknowledge Dr. William D. Hobbs of the Outreach Medical Clinic, Essequibo Coast Guyana, for his invaluable assistance in carrying out this study.

\section{REFERENCES}

1. Martens P, Hall L. Malaria on the move: human population movement and malaria transmission. Emerging Infectious Diseases 2000; 6: 103-109.

2. Roberts DR, Laughlin LL, Hsheih P, Legters LJ. DDT, global strategies, and a malaria control crisis in South America. Emerging Infectious Diseases 1997; 3: 295-302.

3. Foster S. Treatment of malaria outside the formal health services. Journal of Tropical Medicine and Hygiene 1995; 98: 29-34.

4. Vundule C, Mharakurwa S. Knowledge, practices, and perceptions about malaria in rural communities of Zimbabwe: relevance to malaria control. Bulletin of the WHO 1996; 74: 55-60.

5. Yeneneh H, Gyorkos TW, Joseph L, et al. Antimalarial drug utilization by women in Ethiopia: a knowledge-attitudespractice study. Bulletin of the WHO 1993; 71: 763-772.

6. McCombie SC. Treatment seeking for malaria: a review of recent research. Social Science and Medicine 1996; 43: 933-945.

7. Lipowsky R, Kroeger A, Vazquez ML. Sociomedical aspects of malaria control in Columbia. Social Science and Medicine 1992; 34: 625-637.

8. Foster S. Pricing, distribution, and use of antimalarial drugs. Bulletin of the WHO 1991; 69: 349-363.

9. Okonofua FE, Feyisetan BJ, Davies-Adetugbo A, Sanusi YO. Influence of socioeconomic factors on the treatment and prevention of malaria in pregnant and non-pregnant adolescent girls in Nigeria. Journal of Tropical Medicine and Hygiene 1992; 95: 309-315.

10. Snow RW, Peshu N, Forster D, et al. The role of shops in the treatment and prevention of childhood malaria on the coast of Kenya. Transactions of the Royal Society of Tropical Medicine and Hygiene 1992; 86: 237-239.

11. Ruebush TK, Zeissig R, Klein RE, Godoy HA. Community participation in malaria surveillance and treatment II. American Journal of Tropical Medicine and Hygiene 1992; 46: 261-271.

12. Ruebush TK, Weller SC, Klein RE. Knowledge and beliefs about malaria on the Pacific coastal plain of Guatemala. American Journal of Tropical Medicine and Hygiene 1992; 46: 451-459.

13. Haak H. Pharmaceuticals in two Brazilian villages: lay practices and perceptions. Social Science and Medicine 1988; 27: 1415-1427.

14. Igun UA. Why we seek treatment here: retail pharmacy and clinical practice in Maiduguri, Nigeria. Social Science and Medicine 1987; 24: 689-695.

15. Deming MS, Gayibor A, Murphy K, et al. Home treatment of febrile children with antimalarial drugs in Togo. Bulletin of the WHO 1989; 67: 695-700.

16. Mwabu GM. Health care decisions at the household level: results of a rural health survey in Kenya. Social Science and Medicine 1986; 22: 315-319.

17. Gomes M, Wayling S, Pang L. Interventions to improve the use of antimalarials in South-East Asia: an overview. Bulletin of the WHO 1998; 76(Suppl.1): 9-19.

18. Foster S. Economics and its contribution to the fight against malaria. Annals of Tropical Medicine and Parasitology 1998; 92: 391-398.

19. Foster S. Supply and use of essential drugs in Sub-Saharan Africa: some issues and possible solutions. Social Science and Medicine 1991; 32: 1201-1218.

20. Pan American Health Organization. Health conditions in the Americas: 1998 edition. Washington DC: PAHO; 1998.

21. Pan American Health Organization. Health conditions in the Americas: 1996 edition. Washington DC: PAHO; 1996.

22. Rambajan I. Highly prevalent falciparum malaria in north-west Guyana: its development, history and control problems. Bulletin of the PAHO 1993; 28: 193-201.

23. Department of Communicable Diseases, Ministry of Health Guyana. Malaria in Guyana; 1998. (Preliminary report, unpublished).

24. Rawlins SC. The malaria situation in the Caribbean region. West Indian Medical Journal 1993; 42: 134-136. 
25. World Health Organization. World malaria situation in 1989 Weekly Epidemiological Record 1991; 22: 162

26. Phillips EJ, Keystone JS, Kain KC. Failure of combined chloroquine and high-dose primaquine therapy for Plasmodium vivax malaria acquired in Guyana, South America. Clinical Infectious Diseases 1996; 23: 1171-1173.

27. Klein RE, Weller SC, Zeissig R, et al. Knowledge, beliefs, and practices in relation to malaria transmission and vector control in Guatemala. American Journal of Tropical Medicine and Hygiene 1995; 52: 383-388.

28. IMF Statistics Department. International Financial Statistics Yearbook Vol. LII. Washington DC: International Monetary Fund; 1999.

29. Fungladda W, Honrado ER, Thimasarn K, et al. Compliance with artesunate and quinine + tetracycline treatment of uncomplicated $P$. falciparum malaria in Thailand. Bulletin of the WHO 1998; 76: 59-66.
30. Denis MB. Improving compliance with quinine + tetracycline for treatment of malaria: evaluation of health education interventions in Cambodian villages. Bulletin of the WHO 1998; 76(Suppl.1): 43-49.

31. White NJ. Why is it that antimalarial drug treatments do not always work? Annals of Tropical Medicine and Parasitology 1998; 92: 449-458.

32. Weinke T, Held T, Trautmann M, et al. Malaria therapy in 452 patients, with special reference to the use of quinine. Journal of Infection 1992; 25: 173-180.

33. Karbwang J, Na-Bangchang K, Thanavibul A, et al. Comparison of oral artesunate and quinine plus tetracycline in acute uncomplicated P. falciparum malaria. Bulletin of the WHO 1994; 72: 233-238.

34. Qingjun L, Jihui D, Laiyi $\mathrm{T}$, et al. The effect of drug packaging on patients' compliance with treatment for Plasmodium vivax malaria in China. Bulletin of the WHO 1998; 76(Suppl.1): 21-27.

Dr. Christopher Booth, M.D., conducted this research while he was a student at Queen's University School of Medicine. $\mathrm{He}$ is now a first year resident in Internal Medicine at the University of Toronto. Dr. Dick MacLean, M.D., FRCPC, is the director of the Centre for Tropical Disease at McGill University. 